\title{
Transnationale Formen der Partizipation in grenzüberschreitenden Räumen
}

\section{Carolin Zwilling}

\section{Einleitung}

Dieser Vortrag widmet sich einem bislang ${ }^{1}$ aus rechtlicher Sicht kaum untersuchten Themengebiet. Sowohl die Partizipationsformen direkter und deliberativer Demokratie als auch die Entwicklung grenzüberschreitender Räume sind jeweils als Einzelthemen ausführlich und im Rahmen verschiedener Fachrichtungen wissenschaftlich diskutiert worden. Die Kombination beider Themen hingegen wirft neue Fragestellungen auf, die besonders aus verfassungsrechtlicher Sicht einer weitergehenden Analyse bedürfen.

Kern dieser Analyse sind die zentralen Begriffe „Partizipationsformen“, "grenzüberschreitende Räume“ sowie das sie miteinander auf innovative Weise verbindende Adjektiv „transnational“.

Ein näherer Blick auf Europas Grenzregionen legt nahe, dass diese als Laboratorien für den europäischen Integrationsprozess dienen, indem sie nicht nur der Ort für eine enge Kooperation zwischen Verwaltungsbehörden verschiedener Staaten sind (top-down), sondern auch den am besten geeigneten Ort für grenzüberschreitende Bürgerbeteiligung (bottomup) darstellen können. ${ }^{2}$ Damit kann sich die grenzüberschreitende Zusammenarbeit sowohl als funktionales als auch als demokratisch-partizipatives Instrument erweisen. ${ }^{3}$

1 Es handelt sich um die Verschriftlichung des Vortrages im Rahmen der Konferenz "Governance auf dem Prüfstand: Direkte und Deliberative Demokratie in Europa“ vom 25. bis 27.10.2017.

2 Vgl. Peter Bußjäger und Anna Gamper (Hrsg.), Demokratische Innovation und Partizipation in der Europaregion, (nap, 2015).

3 " $\mathrm{CBC}$ regions are framed as laboratories for European integration and are therefore favorable arenas for both a space for cross-border administrative cooperation and a model for modes of citizens' participation" vgl. Peter Ulrich, »Institutionalizing (cross-border) citizenship on subnational level - The European Grouping of Territorial Cooperation (EGTC) as a new administrative space for participatory and functional governance in Europe«, in Federal Governance (2016) 13, 11ff (31). 


\section{Versuch einer Begriffsbestimmung}

Die zahlreichen Formen direkter und deliberativer Demokratie beruhen auf den jeweiligen nationalen Rechtsordnungen und sind somit üblicherweise in den Staatsverfassungen und Staatsgesetzen geregelt. In föderalen Bundesstaaten oder Regionalstaaten können sie auch in substaatliche Kompetenzbereiche fallen, etwa der Bundesländer ${ }^{4}$ oder Regionen ${ }^{5}$.

Der Begriff der grenzüberschreitenden Zusammenarbeit eröffnet hingegen ein sehr weit gefasstes und interdisziplinäres Forschungsfeld ${ }^{6}$, das im Rahmen dieses Beitrages für eine verfassungsrechtliche Untersuchung auf jene Grenzregionen beschränkt werden muss, die einerseits durch Staatsgrenzen - und somit nicht lediglich innerstaatliche Verwaltungsgrenzen - gekennzeichnet sind und zudem bereits ein Mindestmaß an grenzüberschreitender Kooperation aufweisen. Diese Kooperation kann sowohl die Zusammenarbeit von lokalen und regionalen Gebietskörperschaften wie auch jene im non-gouvernementalen Bereich umfassen. So ist etwa der regelmäßige Austausch zwischen wirtschaftlichen und zivilgesellschaftlichen Akteuren oftmals Auslöser für langfristige und in wachsendem Maße institutionalisierter Kooperation innerhalb eines homogenen Grenzgebietes. Somit umfasst gemäß eines weiten Begriffsverständnisses die grenzüberschreitende Zusammenarbeit jede Aktivität, die beabsichtigt, Ressourcen von mindestens zwei Akteuren aus unterschiedlichen Staaten auf die Erreichung von gemeinsam definierten Zielen auszurichten. ${ }^{7}$ Dabei kennzeichnen vier Dimensionen ${ }^{8}$ die Kooperation: räumliche Merkmale wie

4 Für einen internationalen Überblick vgl. Peter Bußjäger, Alexander Balthasar und Niklas Sonntag (Hrsg.), Direkte Demokratie in Tirol, (nap, 2014).

5 Siehe als Beispiel für die Autonome Provinz Bozen Esther Happacher, »Rechtliche Formen und Voraussetzungen direkter Demokratie in Region und autonomer Provinz: Aktuelle Entwicklungen am Beispiel Südtirols«, in Peter Bußjäger und Anna Gamper (Fn. 2), 47ff.

6 Vgl. Peter Schmitt-Egner, »Grundlagen, Bestand und Modelle grenzüberschreitender Zusammenarbeit in den sechs Gründerstaaten der EG am Beispiel der Transnationalen Regionen Saar-Lor-Lux-Rheinland-Pfalz-Wallonien«, in Ulrich Hufeld, Peter-Christian Müller-Graf, Stefan Okruch (Hrsg.), Nachbarschaften innerhalb der Europäischen Union, (Nomos, 2008), 83ff.

7 Vgl. Sara Svensson und Peter Balogh, "Limits to Integration: Persisting Border Obstacles in the EU«, in Eduardo Medeiros (Hrsg.), European Territorial Cooperation. Theoretical and Empirical Approaches tot he Process and Impacts of Cross-Border and Transnational Cooperation in Europe, (Springer, 2018), $115 \mathrm{ff}$.

8 Vgl. Frédéric Durand, »Theoretical Framework of the cross-border space production - the case of the Eurometropolis Lille-Kortrijk-Tournai«, (EUBORDERSCAPES Working Paper 9, 2015), 11f. 
wirtschaftliche oder soziale Faktoren einer Grenzregion bestimmen die strukturelle Dimension des gemeinsamen Raumes, der zweitens durch jegliche Art des Austausches ebenso eine funktionale Dimension erhält. Eine strukturierte Vernetzung von Akteuren, die gemeinsame Steuerungsmechanismen wie Arbeitsgruppen oder regionale Räte bilden, eröffnet eine institutionelle Dimension der Kooperation. Letztendlich bedarf es für den vorliegenden Beitrag auch der vierten Dimension, nämlich der ideellen Verbindung aufgrund von gemeinsamen sozialen, politischen und kulturellen Wertvorstellungen, die eine enge Bindung des Bürgers an den grenzüberschreitenden Raum schaffen.

Das bedeutet zusammenfassend, dass die Kooperation von bloßem Informationsaustausch zwischen non-gouvernementalen Akteuren bis hin zu abgestimmtem politischen Verhalten der öffentlichen Entscheidungsträger reichen kann. Dabei können jeweils sehr unterschiedliche Intensitätsstufen erreicht werden, bis hin zu formalisierten und rechtlich komplexen Kooperationsformen. Sogenannte „cross-border governance bodies“ können auf völkerrechtlichen bilateralen Staatsabkommen oder auch Europäischem Unionsrecht beruhen. Bekannte Beispiele sind die institutionalisierten Formen durch das Madrider Rahmenübereinkommen und seiner $\mathrm{Zu}$ satzprotokolle ${ }^{9}$ sowie der Europäische Verbund territorialer Zusammenarbeit ${ }^{10}$.

Der Begriff „transnationale“ Formen der Partizipation möchte in diesem Beitrag darauf hinweisen, dass aber nicht jene Formen direkter und deliberativer Demokratie gemeint sind, die lediglich in einem der Teilgebiete des grenzüberschreitenden Raumes nach dort geltendem staatlichen Recht angewandt werden und damit lediglich lokal begrenzt sind, auch wenn sie vielleicht allen dort Ansässigen oder den Unionsbürgern offen stehen. ${ }^{11}$ Die Partizipationsform selbst soll als transnational definiert werden können. Peter Ulrich definiert partizipative Governance als self-government einer Vielzahl von Akteuren in einem grenzüberschreitenden

9 Europarat, Europäisches Rahmenabkommen über die grenzüberschreitende Zusammenarbeit zwischen Gebietskörperschaften, SEV-Nr. 106, in Kraft getreten im Dezember 1981.

10 Verordnung (EG) Nr. 1082/2006 des Europäischen Parlaments und des Rates vom 5.7.2006 über den Verbund für territoriale Zusammenarbeit (EVTZ), Amtsblatt Nr. L 210 vom 31.7.2006 S. 0019-0024, in Kraft getreten am 1.8.2006.

11 Für ausführliche Beispiele vgl. Peter Bußjäger, Alexander Balthasar und Niklas Sonntag (Fn. 4). 
transnationalen Raum als Antwort auf Defizite in der nationalen Koordinierung. ${ }^{12}$

Daraus folgt aber verfassungsrechtlich, dass zwischen den gesetzesschaffenden Formen (im Sinne einer Volksgesetzgebung) und reinen konsultativen Formen vor oder bei Gesetzgebungsprozessen unterschieden werden muss. Ob und, wenn ja, inwiefern ein Bevölkerungsteil eines anderen Staates bei der Ausübung der Gesetzgebung als Souverän einbezogen werden könnte, erscheint grundsätzlich schon fraglich. Hervorzuheben ist außerdem, dass eine originäre Gesetzgebungskompetenz eines der oben genannten „cross-border governance bodies“ derzeit ausgeschlossen ist. Eine Form von grenzüberschreitender Governance und Politikgestaltung unterliegt jedenfalls zwei grundlegenden Voraussetzungen: Zum einen muss politische Verantwortlichkeit eindeutig zuordnebar sein und damit eine Kontrollmöglichkeit für das politische Handeln bestehen, zum anderen den politischen Rückhalt und damit letztendlich Legitimität für das Handeln aus der gesamten grenzüberschreitenden Bevölkerung erhalten. Nur eine kontinuierliche Rückkoppelung an den Bürgerwillen aller Teilgebiete des Grenzraumes schafft das notwendige Vertrauen in die Entscheidungsträger. Erst in einem zweiten Schritt entsteht als Folge des Vertrauens das Zugehörigkeitsgefühl aller Bevölkerungsteile zu dem gemeinsamen Raum. Nur in einem solchen Fall übernimmt man freiwillig Pflichten, denn man erkennt zugleich, dass diese Pflichten durch die individuellen Vorteile, die der gemeinsame Raum jedem bietet, ausgeglichen werden. Eine grenzübergreifende Governance, die Synergien zwischen allen beteiligten Akteuren schafft und über die ausschließliche Förderung von Kooperation zwischen öffentlichen Verwaltungen hinausgeht, garantiert die Fortentwicklung eines Grenzraumes am ehesten. ${ }^{13}$

\section{Welche Formen grenzüberschreitender Partizipation sind überbaupt möglich?}

Die nationalen Rechtsordnungen setzen Schranken, verfassungsrechtliche wie einfachgesetzliche, für die konkrete Ausgestaltung der Bürgerbeteili-

12 Ulrich, »Institutionalizing (Fn. 3), 19.

13 Vgl. Julia Boman und Eiki Berg, »Identity and Institutions Shaping Cross-Border Cooperation at the Margins of the European Union«, (Regional \& Federal Studies 2007), 195ff. Zu den institutionellen Merkmalen vgl. Alice Engl, »Partizipative Governance und Mehrebenen-Governance in grenzüberschreitenden Kontexten: Ausgewählte EVTZ-Beispiele im Vergleich«, in Peter Bußjäger und Anna Gamper (Fn. 2), $123 \mathrm{ff}$. 
gungsformen. Die Geltung der jeweiligen Regelung endet an der Staatsgrenze. Mithin erscheint bereits auf den ersten Blick das aufeinander abgestimmte Verhalten in allen beteiligten Gebietskörperschaften als einzig gangbarer Weg. Als Beispiel kann hier das Thesenpapier "Fortschritt in der Europaregion Tirol - Südtirol - Trentino" dienen, das in seiner Forderung „Euroregionale Stärkung der Bürgerinnen- und Bürgerbeteiligung, der direkten Demokratie und der Zivilgesellschaft" Folgendes hervorhebt: "Jeder Gebrauch der Instrumente direkter oder partizipativer Demokratie muss die ihnen durch die nationalen Verfassungen gezogenen Grenzen im Blick haben. Eine grenzüberschreitende (...) Anwendung von Instrumenten der direkten Demokratie können (...) nur durch ein abgestimmtes Verhalten der beteiligten Gebietskörperschaften hergestellt werden. "14 Wie bereits festgestellt besteht derzeit keine originäre Gesetzgebungskompetenz eines „cross-border governance body“. Daher kann das Ziel der vielen Initiativen der grenzüberschreitenden Kooperation nur die bestmögliche Anwendung der bereits bestehenden Kompetenzen sein. ${ }^{15}$

Grenzüberschreitende transnationale Partizipation von Bürgern ist im europäischen Mehrebenensystem grundsätzlich auf Ebene der Europäischen Union, auf Länderebene in föderalen Systemen oder Regionen in Regionalstaaten sowie auf kommunaler Ebene denkbar.

Auf europäischer Ebene könnte an die Bürgerbeteiligung mittels Referenden etwa im Rahmen eines Beitrittsverfahrens zur Union oder auch zur Ratifizierung eines neuen Vertrages im Rahmen eines Abänderungsverfahrens wie zuletzt des Lissabonvertrages gedacht werden. Hier mangelt es jedoch am grenzüberschreitenden Charakter, da es sich stets um nationale Referenden im Hinblick auf die eigene Mitgliedschaft in der Europäischen Union handelt. Ebenso wenig bietet sich die Europäische Bürgerbeteiligung als Instrument an, die sich nicht auf einen eng begrenzten Grenzraum für die nur dort ansässige Bevölkerung nutzen lässt.

Da sich keine Rechtsinstrumente im Unionsrecht finden lassen, die transnationale Partizipationsformen begründen würden, hat die Europäische Kommission eine Reihe von EU Bürgerdialogen in zahlreichen Grenzregionen initiiert. ${ }^{16}$ Diese bieten den Unionsbürgern untereinander sowie mit Persönlichkeiten aus der Politik die Möglichkeit, Themen ihres

14 Thesenpapier "Fortschritt in der Europaregion Tirol - Südtirol - Trentino", Euregio-Lab 2014, Punkt V, 8f, abrufbar unter www.europaregion.info/de.

15 Istituto di Sociologia Internazionale di Gorizia (Hrsg.), European Experience of Citizens' Participation in Cross-border Governance (online 2015), 6.

16 Siehe ausführliche Informationen unter https:/ec.europa.eu/info/events/citizens-d ialogues. 
Grenzraumes und auch die gesamte EU betreffend zu diskutieren. Eine Umfrage der Bertelsmann Stiftung zeigt eine sehr hohe Zufriedenheit unter den Teilnehmern. ${ }^{17}$ Dennoch ist zu unterstreichen, dass es sich zwar um eine transnationale Form der Partizipation handelt, diese jedoch nicht gezielt für den Willensbildungsprozess oder auch nur für die Vorbereitung eines Gesetzesaktes angewandt wird. Es handelt sich allein um Diskussionsforen.

Auf substaatlicher Ebene ist der Blick vor allem dorthin zu lenken, wo Bundesländer oder Regionen mit Gesetzgebungskompetenzen etwa im Rahmen von sog. Europaregionen grenzüberschreitende Zusammenarbeit fördern. Der Begriff Euregio oder Europaregion ist nicht juristisch bestimmt und kann unterschiedliche Intensitätsstufen der Kooperation umfassen. In institutionalisierten Kooperationsformen mit rechtlich komplexen Regelungen wie etwa dem Europäischen Verbund für territoriale Zusammenarbeit (EVTZ) sind diese nicht nur theorethisch denkbar, sondern auch in der Praxis in Ansätzen in Anwendung. Zu verzeichnen ist eine deutliche Tendenz zu mehr Partizipationsansätzen und möglicher Teilnahme durch die Bevölkerung am politischen Willensbildungsprozess je komplexer die Struktur der institutionalisierten Kooperationsform ist. ${ }^{18}$

Zunächst ist hier zwischen Partizipationsformen in der Governance Struktur einerseits und den Formen von grenzüberschreitender Bürgerbeteiligung andererseits zu unterscheiden. $\mathrm{Zu}$ ersterem gehören etwa Kooperationsgremien im institutionellen Aufbau von Europäischen Verbünden für territoriale Zusammenarbeit. Derartige Gremien garantieren die Verknüpfung zwischen den verschiedenen Ebenen eines transnationalen Handlungsraumes und bieten damit den regelmäßigen Austausch zwischen den öffentlichen Akteuren (politische Institutionen oder Verwaltungsapparat) und den nicht-öffentlichen Akteuren (Zivilgesellschaft). ${ }^{19}$ Die Mitglieder des Gremiums, die die Bürgerschaft repräsentieren, sind jedoch nur als Individuen dorthin berufen und können nicht die Rückkoppelung an die Gesamtbevölkerung des grenzüberschreitenden Raumes garantieren. Als Beispiel für ein Gremium mit Bürgerbeteiligung kann der EVTZ GO ${ }^{20}$ dienen, bei dem Bürgerdelegierte in "Ständigen Komitees“ in der Ausarbeitungsphase von Projektvorhaben teilnehmen, jedoch nach Abschluß der Planungsphase bei der Entscheidungsfindung und Abstim-

17 Bertelsmann Stiftung (Hrsg.), Evaluation of the Cross-Border EU Citizens` Dialogue (online 2019).

18 Ulrich, »Institutionalizing «(Fn. 3), 20.

19 Schmitt-Egner, »Transnationale Handlungsräume« (Fn. 6), $15 \mathrm{ff}$.

$20 \mathrm{Vgl}$. ausführlich unter https://euro-go.eu/en. 
mung ausgeschlossen sind (Co-Drafting aber kein Co-Decision Making). Auch der institutionelle Aufbau des EVTZ Ister-Granum sieht ein sogenanntes „Bürger Parlament“ vor, das letztlich jedoch nur in Form eines Bürgerforums als reines Beratungsorgan fungiert. ${ }^{21}$ Der EVTZ Eurométropole Lille-Kortrijk-Tournai hat institutionell wie finanziell in seine Strukturen sowohl Arbeitsgruppen wie auch das zivilgesellschaftliche Forum eingebunden. ${ }^{22}$ An beiden nehmen nicht-öffentliche und zivilegesellschaftliche Akteure teil, die jedoch im Rahmen des vorliegenden Beitrages lediglich als eine indirekte Bürgerbeteiligung von Ausschnitten der Bevölkerung klassifiziert werden können.

Formen grenzüberschreitender Bürgerbeteiligung können wie oben beschrieben nur auf aufeinander abgestimmtem Verhalten der jeweiligen Teilregionen beruhen. Die gesetzlichen Schranken der nationalen Rechtsordnungen verhindern ein echtes gemeinsames Rechtsinstrument. Denkbar sind parallelisiert abgehaltene Volksbefragungen, die mit derselben Fragestellung und zeitgleich stattfindend den Willen der Gesamtbevölkerung wiedergeben können. Volksbegehren und Volksabstimmungen hingegen wären ausgeschlossen, da nur der jeweilige Bevölkerungsanteil - als Souverän nur im eigenen Hoheitsgebiet - ein Gesetz einfordern bzw. über eine Gesetzesvorlage abstimmen könnte.

Im Rahmen des Petitionsrechts eines jeden Bürgers könnten auch parallelisierte Bürgerinitiativen gestartet werden. Diese beziehen sich üblicherweise auf Verwaltungshandeln und unterscheiden sich dementsprechend von gesetzgeberischer Bürgerbeteiligung. Der Ausgang und die Behandlung einer solchen Initiative durch die jeweiligen staatlichen öffentlichen Akteure wäre getrennt voneinander und den nationalen Verfahrensregeln entsprechend zu untersuchen.

Einen vor allem für Europäische Verbünde territorialer Zusammenarbeit interessanten Weg könnten euroregionale Bürgerräte bieten. Der üblicherweise jährlich von den Mitgliedern des EVTZ bereit gestellte Haushalt könnte zu einem Anteil als Bürgerhaushalt erarbeitet und beschlossen werden. Eine solche Einbindung des Bürgers würde für einen sensiblen und transparenten Umgang mit den Finanzressourcen sorgen und gleichzeitg das Vertrauen schaffen, dass damit etwas für die Betroffenen geleistet würde. Bürgerhaushalte haben sich ausgehend von Lateinamerika in den vergangenen 30 Jahren in unterschiedlichen Formen als Beteiligungsin-

21 Vgl. ausführlich unter http://istergranum.eu/en.

22 Alice Engl, »Partizipative Governance» (Fn. 13), $131 \mathrm{ff}$. 
strument auf lokaler Ebene erfolgreich verbreitet. ${ }^{23}$ Die Bevölkerung erhält so die Möglichkeit, sich bei der Planung und/oder Verteilung öffentlicher Finanzmittel zu beteiligen. Anhand von sechs Idealtypen unterscheidet man zwischen den Typen als Partizipative Demokratie, Bürgernahe Demokratie, Partizipative Modernisierung, Multi-Stakeholder-Partizipation, Neokorporatismus und Community Development. Diese Idealtypen wurden anhand von sechs Kriterien definiert: dem Kontext (sozio-politischer Rahmen), dem normativen Rahmen (Ideologien und politische Ziele), dem Verfahren (Regeln der Bürgerbeteiligung), den Dynamiken kollektiven Handelns (Einfluss der Zivilgesellschaft, Existenz von Bottom-up-Bewegungen etc.), dem Verhältnis zwischen konventioneller Politik und Beteiligung sowie anhand von Stärken, Schwächen und Herausforderungen. ${ }^{24}$ Während sich das Instrument auf lokaler Ebene weltweit ${ }^{25}$ etablieren konnte, besteht weiterhin eine besondere Herausforderung darin, es auf regionale oder nationale Ebene zu übertragen. So hat Italiens Region Latium einen Bürgerhaushalt eingeführt und Portugal sogar einen nationalen Bürgerhaushalt, bei dessen ersten Durchgang 2017 ein Budget von drei Millionen Euro zur Diskussion stand. Für ein transnationales Verfahren jedoch, etwa im Rahmen eines EVTZ, fehlt es derzeit noch an erfolgreichen Beispielen.

Wenn die Intensität der Einbeziehung des Bürgers in jedem der Landesteile des gemeinsamen Grenzraumes und damit die Nachhaltigkeit seiner Einflussnahmemöglichkeit betrachtet wird, könnte man folgende drei Gruppen bilden, in die sich die oben skizzierten Beispiele einfügen.

Erstens: Eine echte direkte Entscheidung durch alle Bürger gemeinsam und mit Rechtswirkung im gesamten grenzüberschreitenden Raum scheidet vor allem aus verfassungsrechtlichen Gründen aus.

Zweitens: Eine echte gemeinschaftliche Entscheidung, bei der die Bürger gleichrangig neben den Organen der repräsentativen Demokratie mitentscheiden ${ }^{26}$, ist auf grenzüberschreitender lokaler Ebene ebenfalls bislang nicht ersichtlich. Ein EVTZ könnte sich hierfür aufgrund seiner

23 Vgl. Yves Sintomer, Anja Röcke und Carsten Herzberg (Hrsg.), Participatory budgeting in Europe. Democracy and public governance (Routledge 2016).

24 Vgl. Carsten Herzberg, Yves Sintomer und Anja Röcke, »Bürgerhaushalte«, in Veit S., Reichard C., Wewer G. (Hrsg.), Handbuch zur Verwaltungsreform (Springer 2019), 496ff.

25 Engagement Global gGmbH (Hrsg.): Bürgerhaushalte weltweit. Dialog Global Schriftenreibe der Servicestelle Kommunen in der einen Welt (BMZ Nr. 25, 2014).

26 Hier könnte von Joint decision-making oder participation gesprochen werden. 
eigenen Rechtspersönlichkeit als „grenzüberschreitendes Demokratielaboratorium" anbieten. ${ }^{27}$

Drittens: Eine auf reiner Zusammenarbeit beruhende Einbindung des Bürgers in allen Landesteilen des Grenzraumes kann entweder in Form der Meinungserhebung oder der Informationsweitergabe von Seiten der Verwaltung an den Bürger erfolgen, beides sowohl in der Planungs- wie in der Umsetzungsphase. ${ }^{28}$

Ein vertiefter Blick sollte auf die Möglichkeiten geworfen werden, die einige EVTZ bieten können. Grenzüberschreitende Governance und Partizipation ist unstrittig dort einfacher zu verwirklichen, wo bereits gleich gelagerte kulturelle Voraussetzungen beiderseits der Staatsgrenzen vorherrschen. ${ }^{29}$ Kulturelle Homogenität fördert eine gemeinsame Identität, die ihrerseits wiederum Voraussetzung ist für eine bottom-up entstehende partizipative Governance ist. Im Zuge des Europäischen Integrationsprozesses bietet das rechtlich komplexe Instrument des EVTZ zur Institutionalisierung von grenzüberschreitender Zusammenarbeit die besten Möglichkeiten, um ein weites Spektrum von Kompetenzen auf subnationaler Ebene auszuüben und gleichzeitig eine staatliche Ausübung auszuschließen. Gerade hierdurch ist ein EVTZ am geeignetsten, um eine transnationale lokale Governance zu erschaffen, sie stetig anzupassen und mit den Bürgern zu modellieren. ${ }^{30}$ Der Europäische Ausschuss der Regionen fordert ausdrücklich alle EVTZ auf, innovative Formen von Bürgerbeteiligung zu entwickeln und anzuwenden, ${ }^{31}$ und sieht den EVTZ als am besten geeignetes Laboratorium, um öffentliche Politiken auf transnationaler subnationaler Ebene zu entwickeln. ${ }^{32}$ Ein Blick in die konkrete Umsetzung zeigt jedoch durchgehend ein ernüchterndes Bild: partizipative Governance findet in der Praxis lediglich anhand einzelner Aktionen statt wie Job Days oder themenbezogener Informationsveranstaltungen, hingegen bleibt eine insti-

27 Juan-Manuel Trillo-Santamaría, „Cross-Border Regions: The Gap Between the Elite's Projects and the People's Awareness. Reflections from the Galícia-North Portugal Euroregion « (Journal of Borderland Studies 2/2014), $257 \mathrm{ff}$.

28 Darunter wären consultation oder information zu verstehen.

29 Ulrich, »Institutionalizing «(Fn. 3), 21.

30 Ulrich, »Institutionalizing « (Fn. 3), 25.

31 Committee of the Regions, Work Programme for Networks and Platforms for the European Groupings of Territorial Cooperation (EGTC), 2015, 4f. Vgl. Christian Gsodam und Alfonso A. Martínez, "New EU Rules for the EGTC: How the Committee of the Regions Shapes Territorial Cooperation in Europe«, in Alice Engl und Carolin Zwilling (Hrsg.), Functional and More? New Potential for the European Grouping of Territorial Cooperation - EGTC (Eurac Book 63, 2014), 39ff (70).

32 Ulrich, »Institutionalizing« (Fn. 3), 26. 
tutionell verankerte kontinuierliche Mitentscheidung durch die Bürger weiterhin reine Theorie.

\section{Abschließende Bewertung: Statt Teilnahme nur Teilhabe? Statt Teilnahme aber Teilhabe!}

Letztlich lassen die skizzierten Beispiele nur einen Schluß zu: In grenzüberschreitenden Räumen wäre zwar theoretisch, nämlich durch die aufeinander abgestimmten Verfahren in jedem der Einzel-Rechtsräume oder der institutionellen Verankerung innerhalb eines EVTZ, auch echte Teilnahme an Entscheidungs- und Rechtssetzungsprozessen für den Bürger möglich, doch fehlt es bislang an der praktischen Umsetzung derart innovativer Wege.

Die Grenzräume bieten sich derzeit jedoch erfolgreich als Laboratorium für Formen der Teilhabe an Entscheidungsprozessen an. Auf europäischer wie lokaler Ebene nimmt eine wachsende Zahl der Bevölkerung an sogenannten „participation“ oder „consultation“ Verfahren teil, um aktiv die Entscheidungsfindung in den für sie lokal bedeutenden Themenbereichen zu beeinflussen. ${ }^{33}$ Die Arten der Teilhabe können sehr unterschiedlich ausgeprägt sein, von reiner Meinungsäußerung zu vorgegebenen Fragestellungen bis hin zu konstruktiven Vorschlägen bei Lösungsfindungsprozessen. Ebenso ist bei der Institutionalisierung des Verfahrens von einzelfallbezogenen bis hin zu komplex geregelten und wiederkehrenden Verfahren zu unterscheiden. Je komplexer die Verfahrensstruktur für eine grenzüberschreitende Teilhabe der Bürger gestaltet ist, so scheint wiederum zu gelten, desto besser und nachhaltiger funktioniert die Einbindung des Bürgers, was seinerseits einen ersten Schritt in Richtung einer grenzüberschreitenden Bürgergesellschaft darstellt.

Die Teilhabe in der Phase, die der eigentlichen Entscheidungsfindung vorangestellt wird, welche ihrerseits meist den repräsentiven Organen vorbehalten bleibt, ist dennoch ein erster Schritt in die richtige Richtung und sollte daher als Chance für eine umfassende transnationale partizipative Teilnahme gewertet werden.

33 Als Beispiele seien die Initiative „Europe Calling“ in der Region Aachen und Ostbelgien genannt. www.europecalling.nl, ebenso wie MOT und cross-border EU Citizens' Dialogue. 